\title{
Electronic Band Structure and Transport Properties of the Cluster Compound
}

\author{
$\mathrm{Ag}_{3} \mathrm{Tl}_{2} \mathrm{Mo15}_{15} \mathrm{Se}_{19}$ \\ Supporting Information \\ Patrick Gougeon $^{1 *}$, Philippe Gall ${ }^{1}$, Rabih Al Rahal Al Orabi ${ }^{1, \$ *}$, Benoit Boucher ${ }^{1}$, \\ Bruno Fontaine ${ }^{1}$, Régis Gautier ${ }^{1}$, Anne Dauscher $^{2}$, Christophe Candolfi $^{2}$, Bertrand Lenoir ${ }^{2}$ \\ ${ }^{1}$ Univ Rennes, CNRS, ISCR UMR 6226, INSA Rennes, ENSC Rennes, F-35000 Rennes, France. \\ ${ }^{2}$ Institut Jean Lamour, UMR 7198 CNRS - Université de Lorraine, 2 allée André Guinier- \\ Campus ARTEM, BP 50840, 54011 Nancy Cedex, France
}

\$ Present addresses: (a) Solvay, Design and Development of Functional Materials Department, Axel'One, 69192 Saint Fons, France and (b) Central Michigan University, Department of Physics, Mt. Pleasant, MI 48859, USA.

*Corresponding authors: patrick.gougeon@univ-rennes1.fr (P. G.), alrah1r@cmich.edu (R. A. R. A. O.)

\section{Content}

Tables S1. Details of the data collection and structural refinement of the PXRD pattern of the $\mathrm{Ag}_{3} \mathrm{Tl}_{2} \mathrm{Mo}_{15} \mathrm{Se}_{19}$ compound.

Table S2. Fractional atomic coordinates, equivalent isotropic displacement parameters $(\AA)$, multiplicity, and site occupancy factors for $\mathrm{Ag}_{3} \mathrm{Tl}_{2} \mathrm{Mo}_{15} \mathrm{Se}_{19}$.

Table S3. Main interatomic distances $(\AA)$ for $\mathrm{Ag}_{3} \mathrm{Tl}_{2} \mathrm{Mo}_{15} \mathrm{Se}_{19}$ determined by Rietveld refinement against the PXRD data. 
Table S1. Details of the data collection and structural refinement of the PXRD pattern of the $\mathrm{Ag}_{3} \mathrm{Tl}_{2} \mathrm{Mo}_{15} \mathrm{Se}_{19}$ compound.

Crystal data

Chemical formula

$\mathrm{Mr}$

Crystal system, space group Temperature $(\mathrm{K})$

a, c $(\AA)$

$\mathrm{V}\left(\AA^{3}\right)$

$\mathrm{Z}$

Radiation type

Specimen shape, size $(\mathrm{mm})$

Data collection

Diffractometer

Specimen mounting

Data collection mode

Scan method

$2 \theta$ values $\left(^{\circ}\right)$

Refinement

$\mathrm{R}$ factors and goodness of fit

No. of data points

No. of parameters
$\mathrm{Ag}_{3} \mathrm{Mo}_{15} \mathrm{Se}_{19} \mathrm{Tl}_{2}$

3671.7

Trigonal, R-3c

293

9.98042 (10), 57.2525 (6)

$4938.82(8)$

6

$\mathrm{Cu} \mathrm{K} \alpha_{1}, \lambda=1.54051 \AA$

Flat sheet, 25 x 25 x 2

Bruker D8 ADVANCE diffractometer

Packed powder pellet

Reflection

Step

$2 \theta \min =82 \theta \max =120.372 \theta$ step $=0.01$

$\mathrm{Rp}=0.103, \mathrm{Rwp}=0.131, \mathrm{Rexp}=0.091$, $\mathrm{R}(\mathrm{F})=0.040, \chi^{2}=2.103$

14256

70 
Table S2. Fractional atomic coordinates, equivalent isotropic displacement parameters $(\AA)$, multiplicity, and site occupancy factors for $\mathrm{Ag}_{3} \mathrm{Tl}_{2} \mathrm{Mo}_{15} \mathrm{Se}_{19}$.

\begin{tabular}{|r|r|r|r|r|r|r|}
\hline Atom & \multicolumn{1}{l|}{ X } & \multicolumn{1}{l|}{ y } & Z & Ueq & Mult. & s.o.f \\
\hline $\mathrm{Ag}$ & $-0.2406(5)$ & $-0.3799(7)$ & $0.4163(2)$ & $0.041(4)$ & 36 & $0.497(3)$ \\
\hline $\mathrm{Tl}$ & 0 & 0 & $0.37889(5)$ & $0.0340(9)$ & 12 & $1.006(3)$ \\
\hline Mo1 & $-0.1629(2)$ & $-0.1459(2)$ & $0.48053(3)$ & $0.0063(5)$ & 36 & 1 \\
\hline Mo2 & $0.1855(2)$ & $-0.3227(3)$ & $0.45601(3)$ & $0.0038(5)$ & 36 & 1 \\
\hline Mo3 & 0.333333 & $-0.1743(3)$ & 0.416667 & $0.0061(8)$ & 18 & 1 \\
\hline Se1 & $-0.0340(3)$ & $-0.3170(3)$ & $0.48226(4)$ & $0.0041(7)$ & 36 & 1 \\
\hline Se2 & $-0.3819(3)$ & $-0.3641(3)$ & $0.45515(4)$ & $0.0055(7)$ & 36 & 1 \\
\hline Se3 & $0.0349(4)$ & -0.333333 & 0.416667 & $0.0065(11)$ & 18 & 1 \\
\hline Se4 & 0 & 0 & $0.44430(7)$ & $0.0064(11)$ & 12 & 1 \\
\hline Se5 & 0.333333 & -0.333333 & $0.49139(7)$ & $0.0065(12)$ & 12 & 1 \\
\hline
\end{tabular}


Table S3. Main interatomic distances (A) for $\mathrm{Ag}_{3} \mathrm{Tl}_{2} \mathrm{Mo}_{15} \mathrm{Se}_{19}$ determined by Rietveld refinement against the PXRD data.

\begin{tabular}{|l|l|}
\hline $\mathrm{Ag}-\mathrm{Se} 2$ & $2.680(11)$ \\
\hline $\mathrm{Ag}-\mathrm{Se} 2$ & $2.725(11)$ \\
\hline $\mathrm{Ag}-\mathrm{Se} 3$ & $2.549(7)$ \\
\hline $\mathrm{Ag}-\mathrm{Se} 4$ & $2.973(9)$ \\
\hline Tl-Se1 (x3) & $3.635(3)$ \\
\hline Tl-Se2 (x3) & $3.289(3)$ \\
\hline Tl-Se4 & $3.745(5)$ \\
\hline Tl-Se5 & $3.101(5)$ \\
\hline Mo1-Mo1 (x2) & $2.681(3)$ \\
\hline Mo1-Mo1 (x2) & $2.714(3)$ \\
\hline Mo1-Mo2 & $3.555(4)$ \\
\hline Mo1-Se1 & $2.583(3)$ \\
\hline Mo1-Se1 & $2.612(5)$ \\
\hline Mo1-Se1 & $2.603(3)$ \\
\hline Mo1-Se2 & $2.621(3)$ \\
\hline Mo1-Se4 & $2.588(4)$ \\
\hline Mo2-Mo2 (x2) & $2.652(4)$ \\
\hline Mo2-Mo3 & $2.694(2)$ \\
\hline Mo2-Mo3 & $2.786(3)$ \\
\hline Mo2-Se1 & $2.681(4)$ \\
\hline Mo2-Se2 & $2.649(4)$ \\
\hline Mo2-Se2 & $2.561(4)$ \\
\hline Mo2-Se3 & $2.680(3)$ \\
\hline Mo2-Se5 & $2.540(4)$ \\
\hline Mo3-Mo3 (x2) & $2.749(4)$ \\
\hline Mo3-Se2 (x2) & $2.628(3)$ \\
\hline Mo3-Se3 (x2) & $2.581(3)$ \\
\hline
\end{tabular}

